\title{
Experimental validation of numerical sensitivities in a deep drawing simulation
}

\author{
A.H. van den Boogaard ${ }^{1}$, B.D. Carleer ${ }^{2}$, E.H. Atzema ${ }^{3}$, E.V. ter Wijlen ${ }^{1}$ \\ ${ }^{1}$ University of Twente - P.O. Box 217, 7500 AE Enschede, Netherlands \\ e-mail:a.h.vandenboogaard@utwente.nl,e.v.terwijlen@student.utwente.nl \\ ${ }^{2}$ Autoform Engineering Deutschland GmbH - Emil Figge Straße 76-80, Dortmund, Germany \\ e-mail: bart.carleer@autoform.de \\ ${ }^{3}$ Corus RD\&T - P.O. Box 10000, 1970 CA IJmuiden, Netherlands \\ e-mail: eisso.atzema@corusgroup.com
}

\begin{abstract}
Deep drawing of a benchmark B-pillar is numerically modelled and experimentally performed with varying blankholder force and several blank shape parameters. The most influential parameters are selected for optimisation. Direct application of Autoform sigma software was used to determine sensitivities, as well as indirect application using response surfaces. Interesting nonlinear sensitivities were found that will be missed with simple linear screening techniques.
\end{abstract}

KEYWORDS: Sheet metal forming, Sensitivity, Robustness

\section{INTRODUCTION}

Sheet metal forming simulations are performed on a daily basis in automotive industry. For optimisation as well as for the prediction of robustness, the sensitivity of results with respect to process and material variation is required. The sensitivity can be predicted from a large number of finite element simulations. To limit the number of simulations it is essential to first determine the most influential parameters. Finally, the computed result depends heavily on the accuracy of the calculated values and their sensitivities.

In order to validate the sensitivities a series of simulations and a series of experiments have been performed while varying identical parameters. Firstly, a series of simulations has been performed to identify the parameters having the most influence on the result of stamping a B-Pillar. Three parameters, the blankholder force and two blank shape parameters turned out to have the most influence. Secondly, a series of experiments has been performed while varying the same parameters. For result evaluation, the thickness and the surface strains have been measured. The trends obtained from simulation and experiment coincided very well although absolute values were sometimes only weakly represented. An unexpected non- linear trend in the obtained safety margin for necking as function of blankholder force could be explained based on the shape of the Forming Limit Curve (FLC) and the strain redistribution.

All FEM calculations in this paper have been performed with Autoform software.

\section{PROBLEM DEFINITION}

This paper considers the optimisation of a typical Bpillar, for which the dies are available for experimental verification. The 7-step methodology for defining an optimisation model by Bonte [1] is used. It consists of the following steps:

1. Determine the appropriate optimisation situation;

2. Select only the necessary responses;

3. Select one response as objective function, the others as implicit constraints;

4. Quantify the objective function and implicit constraints;

5. Select possible design variables;

6. Define the ranges on the design variables;

7. Identify explicit constraints. 
In this case, the dies cannot reasonably be changed and therefore the design variables that can be freely chosen are limited to blank geometry parameters and process settings. Given this constraint, a benchmark optimisation problem was defined in [3]. The optimisation situation was identified as a 'Process design type I' situation, in which process variables are determined, for a fixed product geometry.

The relevant responses (step 2) are: geometrical accuracy (springback), formability (no necking or wrinkling), material and process costs and maximum process force. Effective compensation of shape errors would require die adaptations that were not allowed in this research and costs and maximum force were not limiting the experiments. Therefore necking and wrinkling were considered to be the most important responses.

In step 3, one of these responses should be selected as optimisation target and the other one will automatically be formulated as a constraint. Because necking is clearly recognised in a blank and is a premium reason to reject a product the probability for necking is chosen as the objective function to be minimised, while fulfilling constraints with respect to wrinkling. The quantification of these responses in step 4 use simple strain based measures. The objective is to maximise the minimum distance to the forming limit curve in major strain direction. Prediction of wrinkling is still difficult. In practice, often sheet thickening is used as indicator for wrinkling risk, and that will be used here also. Since a thickness strain of +0.37 was found in a realised acceptable product, the wrinkling constraint was defined as $\varepsilon_{\text {major }}+\varepsilon_{\text {minor }} \geq$ -0.37 , assuming constant volume deformation, although 0.02 is a more customary value for free standing regions.

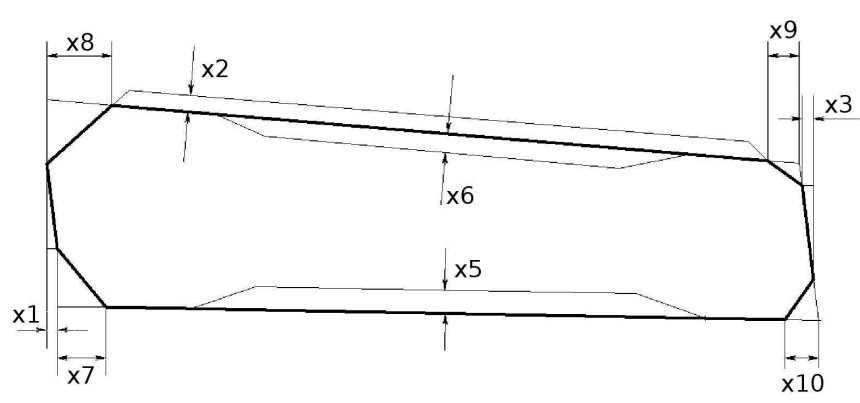

Figure 1: Blank shape design variables

As already indicated, the selection of design variables in step 5 is limited by the experimental constraints.
The tool geometry is fixed and only blank geometry and blankholder force $\left(x_{1}=\mathrm{BHF}\right)$ can be varied. The variables determining the blank geometry are presented in Figure 1. The over all size of the blank is $1500 \times 400 \mathrm{~mm}^{2}$.

Finally design variable ranges (step 6) are defined. No explicit constraints between variables (step 7) are identified. This finally leads to the following optimisation model:

$$
\begin{array}{ll}
\max _{x_{1} \ldots x_{10}} & \left(\min \left(\varepsilon_{\text {major }}-\varepsilon_{\mathrm{flc}}\right)\right) \\
\text { s.t. } & \varepsilon_{\text {major }}+\varepsilon_{\text {minor }} \geq-0.37 \\
& 0 \leq x_{1} \leq 5000 \mathrm{kN} \\
& 0 \leq x_{2}, x_{3}, x_{4}, x_{5}, x_{6} \leq 20 \mathrm{~mm} \\
& 0 \leq x_{7} \leq 80 \mathrm{~mm} \\
& 0 \leq x_{8} \leq 130 \mathrm{~mm} \\
& 0 \leq x_{9}, x_{10} \leq 50 \mathrm{~mm}
\end{array}
$$

For the simulations and the experimental validation in this paper, steel sheet of type H340LAD+Z (EN10292) with a thickness of $1.458 \mathrm{~mm}$ was used. It has a yield stress of $406 \mathrm{MPa}$, a strength of $458 \mathrm{MPa}$ and a hardening exponent $n=0.131$. The $R$-values in $0^{\circ}, 45^{\circ}$ and $90^{\circ}$ directions are respectively 0.891 , 1.037 and 1.053 .

\section{FEM SIMULATIONS}

Preliminary analyses indicated that the risk for wrinkling is highest in region ' $\mathrm{A}$ ' in Figure 2 and the risk for necking is highest in region ' $\mathrm{B}$ '. Therefore, the evaluation of the wrinkling constraint and the objective function was restricted to region $\mathrm{A}$ and $\mathrm{B}$ respectively.

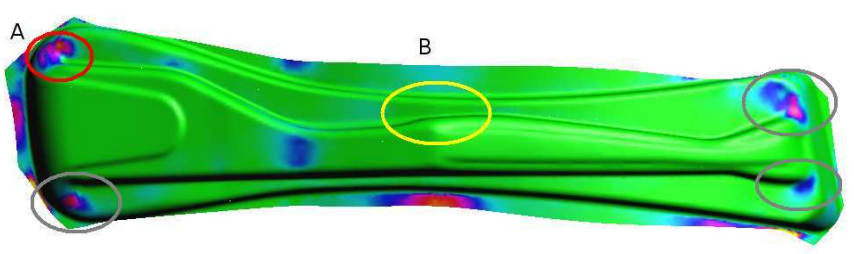

Figure 2: Focus areas for influence analysis

The optimisation model was first reduced by determining the most influential design variables. A resolution III fractional factorial design [2] was applied to the 10 design variables, requiring 16 simulations. The results are presented in Pareto plots for the objective 
function and the wrinkling constraint in Figures 3 and 4 respectively. For both responses, the blankholder force $x_{1}$ has the highest impact. The shape variables $x_{5}$ and $x_{8}$ have a significant influence on either the objective or the constraint function. Therefore, only the influence of variables $x_{1}, x_{5}$ and $x_{8}$ are further investigated.

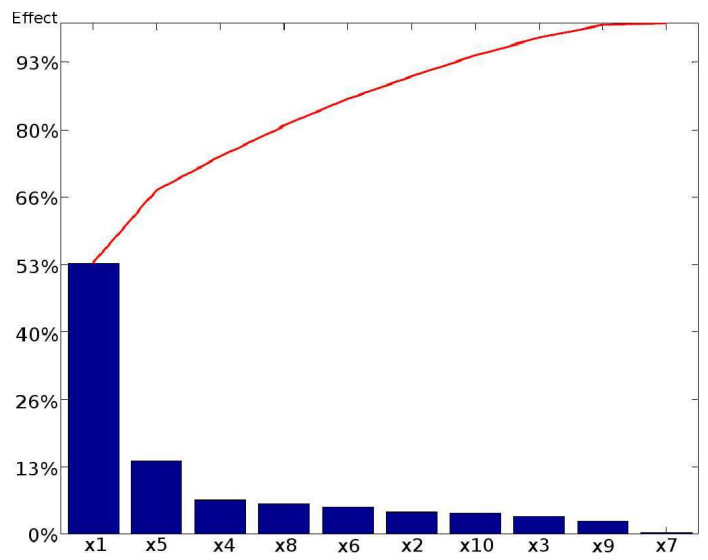

Figure 3: Pareto plot of the influence on the objective

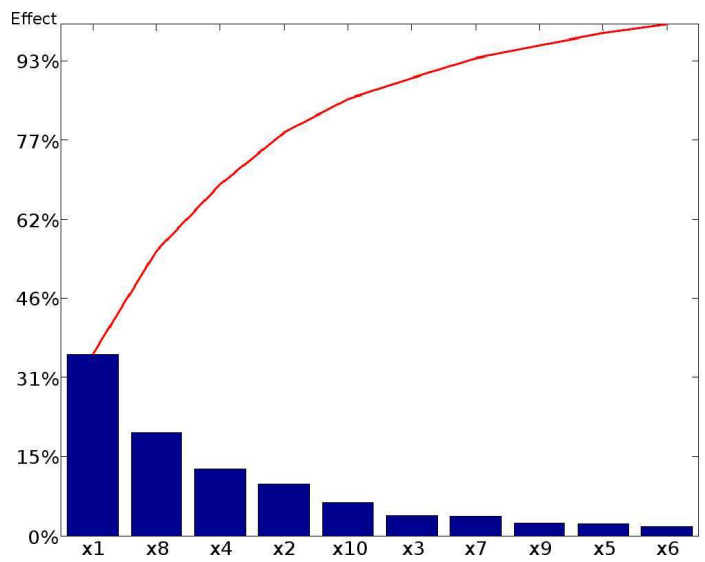

Figure 4: Pareto plot of the influence on the constraint

The selection of these parameters was independently confirmed with an Autoform-sigma analysis, based on 50 calculations.

The scatter plots of necking and wrinkling responses, based on 147 calculations with varying blankholder force are given in Figure 5. As expected, the responses are relatively smooth functions of the blankholder force, until necking commences. From that point on, the major strain becomes highly dependent on the actual place, just inside or outside the necking zone.
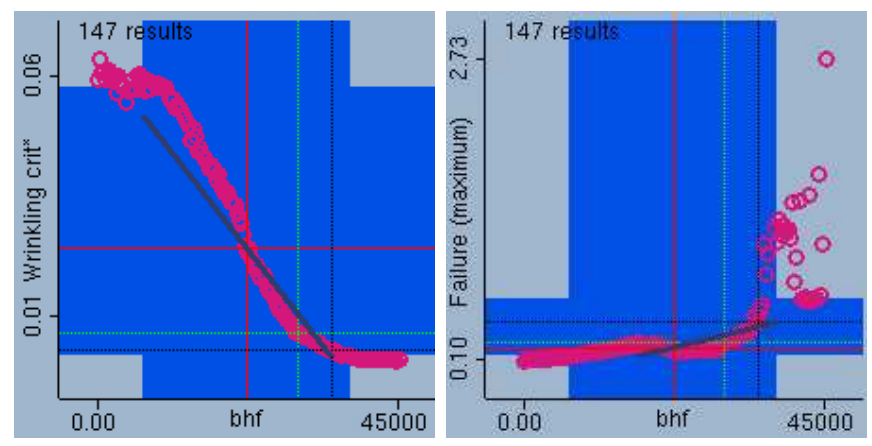

Figure 5: Scatter plots for wrinkling in region A (left) and necking in region $\mathrm{B}$ (right)

At a blank holder force of $2690 \mathrm{kN}$ necking was predicted in region $\mathrm{A}$, while the strains in the expected necking region (B) were still regarded as safe. With further increasing blank holder force necking in region A 'disappeared'. This was discovered with the reference blank shape. In Figure 6 one can see the scatter plot for the failure criterion (necking) in region A.

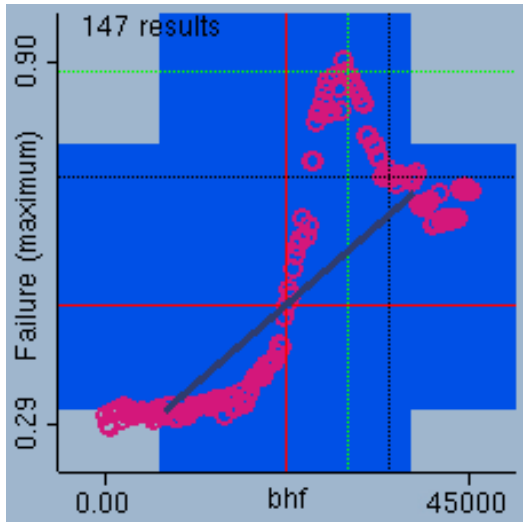

Figure 6: Failure plots of region A.

Note that the maximum value of the failure criterion is still below 1.0, indicating that it is below the FLC. The plotted value, however, is the average strain in region $\mathrm{A}$ and a closer look discovered that 1 element actually exceeded the FLC.

The unexpected intermediate peak (compared to e.g. the regular response in left graph in Figure 5 could partly be explained by the shape of the FLC and a minor strain that increases from negative to positive values. At only slightly increasing major strain, the minimum value of the FLC is passed at $\varepsilon_{\text {minor }}=0$.

In Figure 7 the failure response plot as function of both the blankholder force and shape variable $x_{5}$ is presented. Clearly, there is a strong nonlinear coupling in these two variables. Even at high blankholder 
forces, the risk for necking can be low if a blank cutout at the oposite side of region $\mathrm{B}$ is used.

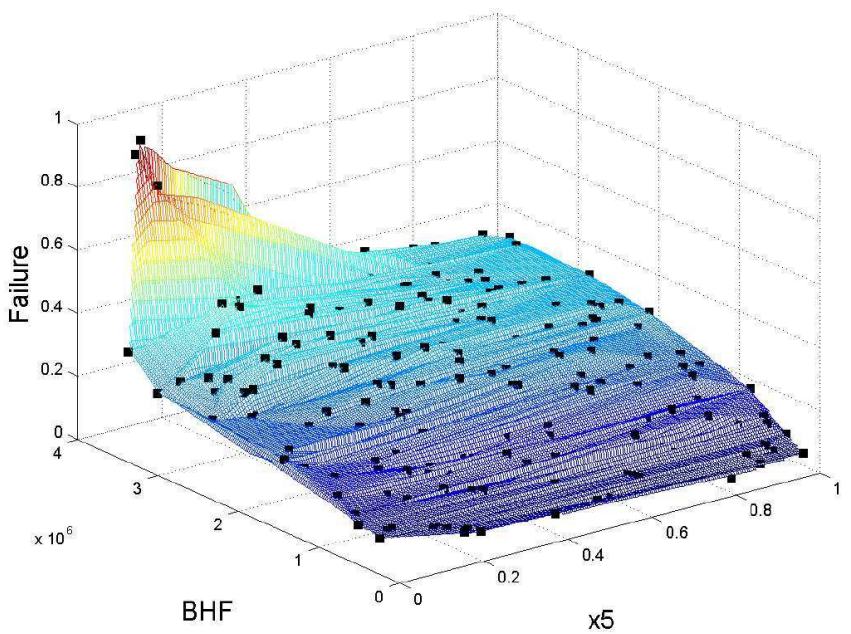

Figure 7: Surface plot of the response failure in region B for $\mathrm{BHF}$ and $x_{5}$.

\section{EXPERIMENTS}

The thicknesses measured in the experiments are presented in Table 1 together with the FEM values. The grayed lines represent experiments showing cracks. In experiment 18 cracks occured only in region $\mathrm{A}$ and in experiment 7 both in region A and B. The finite element simulation predicted necking at a blankholder force of $2690 \mathrm{kN}$ in the same region. The agreement between the experimentally and numerically determined critical blankholder force is very good.

Table 1: Thickness experiments vs FEM

\begin{tabular}{|r|c|c|c|c|c|}
\hline & & \multicolumn{3}{|c|}{ Thickness in mm } \\
Region B & \multicolumn{2}{c|}{ Region A } \\
BHF in kN & Exp num & Exp. & FEM & Exp. & FEM \\
\hline 100 & 8 & 1.417 & 1.335 & 1.507 & 1.446 \\
500 & 9 & 1.374 & 1.323 & 1.477 & 1.440 \\
1000 & 6 & 1.349 & 1.301 & 1.482 & 1.431 \\
1200 & 21 & 1.329 & 1.284 & 1.464 & 1.417 \\
1400 & $20,47,48$ & 1.340 & 1.288 & 1.457 & 1.427 \\
1800 & 19 & 1.331 & 1.271 & 1.464 & 1.419 \\
2200 & 10 & 1.297 & 1.212 & 1.450 & 1.422 \\
2600 & 18 & 1.233 & 1.195 & 1.465 & 1.423 \\
3400 & 7 & 1.252 & 1.151 & 1.431 & 1.407 \\
\hline
\end{tabular}

As one can conclude from Table 1 the thickness in the FEM simulation in region $\mathrm{B}$, is monotonously decreasing and this is also the case in the experiments, apart from the single experiment 21 . This deviating result is attributed to experimental variation. The trend is predicted very well with the FEM simulations, but the thinning itself is somewhat overestimated.

The thickness in the FEM simulation of region $\mathrm{A}$ is not monotonously decreasing with increasing blankholder force. The thinning in region $\mathrm{A}$ is quite low, compared to the initial thickness of $1.458 \mathrm{~mm}$ and the measured variation seems to fall within the experimental noise. The FEM simulation only slightly overestimates the thinning in region A. The measurement of the thickness evolution as function of the blankholder force does not present a clear indication of the occurence of necking, unless accidentally the exact position of a neck is chosen.

\section{CONCLUSIONS}

Analysis of the influence of design variables gives an interesting insight in the deep drawing process conditions. The variation of one parameter at at time can already show significantly non-linear responses, depending on the selected response. This observation makes the application of linear response surfaces for screening purposes awkward.

Surface plots, in which a response is plotted as function of 2 design variables can show significant nonlinear interaction, as shown e.g. for the blankholder force and shape variable $x_{5}$. A weak or even absent sensitivity of $x_{5}$ at a low or intermediate value for BHF may turn out to show a high sensitivity at high BHF. This behaviour will be missed if only scatter plots are used to visualise the process response.

The distance to an FLC is not easily validated experimentally. Thickness measurements can be done more easily. The comparison of experimentally and numerically predicted thicknesses in critical regions as function of the blankholder force showed satisfactory agreements. The trends were predicted with good accuracy.

\section{REFERENCES}

[1] M. H. A. Bonte. Optimisation strategies for metal forming processes. PhD thesis, University of Twente, 2007.

[2] D. Montgomery. Design and analysis of experiments. John Wiley and Sons, Inc., New York, 5 edition, 2001.

[3] E. V. ter Wijlen. Optimisation of a deep drawing process with experimental validation - applied to an automotive deep drawing process of a b-pillar. Master's thesis, University of Twente, 2007. 